\title{
A Parametric Linearization Approach for Solving Zero-One Nonlinear Programming Problems
}

\author{
Asadollah Mahmoodzadeh Vaziri, A. V. Kamyad, S. Efatti \\ Department of Applied Mathematics, Ferdowsi University of Mashhad, Mashhad, Iran \\ E-mail:a_mvaziri@yahoo.com \\ Received March 20, 2011; revised May 29, 2011; accepted June 6, 2011
}

\begin{abstract}
In this paper a new approach for obtaining an approximation global optimum solution of zero-one nonlinear programming (0-1 NP) problem which we call it Parametric Linearization Approach (P.L.A) is proposed. By using this approach the problem is transformed to a sequence of linear programming problems. The approximately solution of the original 0-1 NP problem is obtained based on the optimum values of the objective functions of this sequence of linear programming problems defined by (P.L.A).
\end{abstract}

Keywords: Zero-One Programming, Nonlinear Programming, Nonlinear Optimization

\section{Introduction}

Integer programming is one of the most interesting and difficult research areas in mathematical programming and operations research. During the past years, many works has been devoted to linear integer programming, linear $0-1$ programming and nonlinear 0 -1programming problems [1-4].

Nnonlinear constrained integer programming problem have many applications in sciences and engineering. A number of research paper dealing with reliability optimization problems are reported in the literature. These are integer programming problems with nonlinear separable objective function and nonlinear multi choice constrained $[5,6]$.

A developed optimization method for solving integer nonlinear programming problem (INLP) with $0-1$ variable could be found in [7]. This method is closely related to the lexicographic method of Gilmore and Gomory [8], for the knapsack problem and additive algorithm of Balas [9].

One of the conventional methods for solving zero-one nonlinear programming problem is to transform it to a linear programming problem. The main difficulty of this method is the very large number of variables and constraints which increases the problem-size.

A linearization involving a linear number of variables and constraints was first proposed by Glover [10] and improved by Oral and Kettani $[11,12]$. The resulting linearization involving only $n-1$ additional variable and 2(n-1) linear constraint.

Hanssen and Meyer in [13] compare different ways for linearization the unconstraint quadratic zero-one minimization problem. This approaches involves to increase the number of variables and constraints.

Consider the zero-one nonlinear programming problem as follow:

$$
\left\{\begin{array}{ccc}
\operatorname{minimize} & f(x) & \\
\text { subject to } & g_{s}(x) \leq 0 & s=1, \cdots, m \\
& h_{k}(x)=0 & k=1, \cdots, l \\
& x_{j}=0 \text { or } 1 & j=1, \cdots, n,
\end{array}\right.
$$

where $f():. \mathbb{R}^{n} \rightarrow \mathbb{R}$ and $g_{s}():. \mathbb{R}^{n} \rightarrow \mathbb{R} ; s=1, \cdots, m$ and $h_{k}():. \mathbb{R}^{n} \rightarrow \mathbb{R} ; k=1, \cdots, l$ are nonlinear functions.

We can replace the discrete constraints of the form $x_{j}=0$ or $1 ; j=1, \cdots, n$, by the continuous new ones of the form $x_{j}^{2}-x_{j}=0 ; j=1, \cdots, n$. It is trivial that this two constraints are equivalent. At first we consider zero-one nonlinear programming problem in which the discrete constraint are replaced with continuous ones. Then we have the following nonlinear programming problem:

$$
\left\{\begin{array}{ccc}
\operatorname{minimize} & f(x) & \\
\text { subject to } & g_{s}(x) \leq 0 & s=1, \cdots, m \\
& h_{k}(x)=0 & k=1, \cdots, l \\
& x_{j}^{2}-x_{j}=0 & j=1, \cdots, n,
\end{array}\right.
$$

In this paper we present a new approach call it parametric linearization approach for finding the approxi- 
mated global optimum of zero-one NP problem by solving a sequence of linear programming problems over sub-regions. The solution of the sequence of LP problems tends to the optimal solution of the original nonlinear problem. The reminder of the paper is organized as follow:

The second section contains a description of parametric linearization approximation and the convergence results. The third section contain a description of using the parametric linearization approximation for solving zero-one nonlinear programming problem. In the forth section the approach will be followed to decrease the number of sub-problems which must be solved in each iteration. Numerical examples used to illustrate the efficiency of the proposed approach and they are given in fifth section. The last section draws overall conclusions.

\section{The Parametric Linearization Approach}

Let $f(x)$ be a nonlinear smooth function on $[a, b]$. We know that the linear Taylor expansion of $f(x)$ at the point $x_{0} \in[a, b]$ as follows:

$$
f(x)=f\left(x_{0}\right)+\left(x-x_{0}\right) f^{\prime}\left(x_{0}\right),
$$

where $x_{0}$ be an arbitrary point in $(a, b)$. In usual Taylor expansion $x_{0}$ is a fixed point but in our definition this is an arbitrary point. Thus we may call it moving linear Taylor expansion of $f(x)$.

Definition 2.1: We consider a partition of an interval $[a, b]$ as the following form:

$$
P_{r}[a, b]=\left\{a=y_{0}, y_{1}, \cdots, y_{r}=b\right\},
$$

where $y_{0}<y_{1}<\cdots<y_{r}$.

The norm of partition defined by:

$$
\left\|P_{r}[a, b]\right\|=\max _{0 \leq i \leq r-1}\left\{y_{i+1}-y_{i}\right\} .
$$

Definition 2.2: Let $f(x)$ is a nonlinear function on $[a, b]$ and $P_{r}([a, b])$ be the partition of it. Let defined $f_{i}(x)$ a parametric linear approximation of $f(x)$ on sub-interval $\left[y_{i}, y_{i+1}\right]$ as follow:

$$
\begin{gathered}
f_{i}(x) \triangleq f^{\prime}\left(s_{i}\right) x+f\left(s_{i}\right)-s_{i} f^{\prime}\left(s_{i}\right) \\
x \in\left[y_{i}, y_{i+1}\right] \& i=0, \cdots, r-1,
\end{gathered}
$$

where $s_{i} \in\left(y_{i}, y_{i+1}\right)$ is an arbitrary point.

Now we define $G_{r}(x)$ as the following form:

$$
G_{r}(x)=\sum_{i=0}^{r-1}\left[f_{i}(x) \chi_{\left[y_{i}, y_{i+1}\right]}(x)\right],
$$

where $\chi_{A}(x)$ is the characteristic function and defined as the following:

$$
\chi_{A}(x)= \begin{cases}1 & x \in A \\ 0 & x \notin A\end{cases}
$$

We call $G_{r}($.$) as a parametric piecewise linear ap-$ proximation of $f(x)$ on $[a, b]$.

Note 2.1: In the following theorems it is shown that when the norm of partition tends to zero it means $\left\|P_{r}([a, b])\right\| \rightarrow 0$ then $G_{r}(x)$ is uniformly convergent to $f(x)$ (the original nonlinear function). In the other word we may shown that:

$$
G_{r} \rightarrow f \text { uniformly on }[a, b]
$$

First we show that $G_{r} \rightarrow f$ pointwise on $[a, b]$.

Lemma 2.1: Let $P_{r}([a, b])=\left\{a=y_{0}, y_{1}, \cdots, y_{r-1}, y_{r}=b\right\}$ is an arbitrary partition of $[a, b]$. If $f(y)$ is continuous function on $[a, b]$ and $x, s \in\left[y_{i}, y_{i+1}\right]$ then :

$$
\lim _{\left\|P_{r}\right\| \rightarrow 0}\left(f_{i}(s)+f_{i}^{\prime}(s)(x-s)\right)=f\left(y_{i}\right) .
$$

Proof: The proof is a simple conclusion of the definition.

Definition 2.3: A family $\mathcal{F}$ of complex function $f$ defined on the set $A$ in a metric space $X$ is said to be equicontinuous on $A$ if for every $\varepsilon>0$ there exist $\delta>0$ such that $|f(x)-f(z)|<\varepsilon$ whenever $d(x, z)<\delta$, $x, z \in A, \quad f \in \mathcal{F}$.

Here $d(x, z)$ denote the metric of $A$ (see [6]).

Since $\left\{G_{r}(x)\right\}$ is a sequence of linear function it is trivial that this sequence is equicontinuous.

Theorem 2.1: Let $\left\{f_{N}\right\}$ be an equicontinuous sequence of functions on a compact set $A$ and $\left\{f_{N}\right\}$ converges point-wise on $A$. Then $\left\{f_{N}\right\}$ converge uniformly on $A$.

Proof: Since $\left\{f_{N}\right\}$ is equicontinuous on $A$ then:

$$
\begin{gathered}
\forall \varepsilon>0 \exists \delta>0 \text { s.t.d }(x, z)<\delta \rightarrow\left|f_{N}(x)-f_{N}(z)\right|<\varepsilon \\
x, z \in A, N=1,2, \cdots .
\end{gathered}
$$

It is trivial that for each $x \in A$ and $\delta>0$ we have $A \subseteq \bigcup_{x \in A} N(x, \delta)$. So $\bigcup_{x \in A} N(x, \delta)$ is an open-covering for $A$. Since $A$ is a compact set this open-covering have a finite sub-covering. Thus there exist the point $\alpha_{1}, \alpha_{2}, \cdots, \alpha_{r}$ in $A$ such that $A \subseteq \bigcup_{i=1}^{r} N\left(\alpha_{i}, \delta\right)$. Therefore for each $x \in A$ there exist $\alpha_{i}(i=1,2, \cdots, r)$ in $A$ such that $d\left(x, \alpha_{i}\right)<\delta$ and therefore:

$$
\left|f_{N}(x)-f_{N}\left(\alpha_{i}\right)\right|<\varepsilon \text { for } N=1,2, \cdots .
$$

We know that $\left\{f_{N}\right\}$ is pointwise convergence sequence then there existing a natural number $M$ such that for each $p \geq M, q \geq M$ we have:

$$
\begin{gathered}
\left|f_{q}\left(\alpha_{1}\right)-f_{p}\left(\alpha_{1}\right)\right| \leq \varepsilon \\
\left|f_{q}\left(\alpha_{2}\right)-f_{p}\left(\alpha_{2}\right)\right| \leq \varepsilon \\
\vdots \\
\left|f_{q}\left(\alpha_{r}\right)-f_{p}\left(\alpha_{r}\right)\right| \leq \varepsilon
\end{gathered}
$$

Therefore we have the following inequalities: 


$$
\begin{aligned}
\left|f_{q}(x)-f_{p}(x)\right| & =\left|f_{q}(x)-f_{q}\left(\alpha_{i}\right)+f_{q}\left(\alpha_{i}\right)-f_{p}\left(\alpha_{i}\right)+f_{p}\left(\alpha_{i}\right)-f_{p}(x)\right| \\
& \leq\left|f_{q}(x)-f_{q}\left(\alpha_{i}\right)\right|+\left|f_{q}\left(\alpha_{i}\right)-f_{p}\left(\alpha_{i}\right)\right|+\left|f_{p}\left(\alpha_{i}\right)-f_{p}(x)\right| \leq 3 \eta
\end{aligned}
$$

Then according to the theorem 7.8 in [6] the sequence $\left\{f_{N}\right\}$ is uniformly continuous on $A$ and the proof is completed.

Theorem 2.2: Let $G_{r}(x)$ is a piecewise linear approximation of $f(x)$ on $[a, b]$ as the following form:

$$
G_{r}(x)=\sum_{i=0}^{r-1}\left[f_{i}(x) \chi_{\left[y_{i}, y_{i+1}\right]}(x)\right] .
$$

Then:

$$
G_{r} \rightarrow f \text { uniformly on }[a, b] .
$$

Proof: The proof is immediate consequence of lemma 2.1 and theorem 2.1.

Let $f():. A \rightarrow \mathbb{R}$ is nonlinear smooth function where $A=\prod_{i=1}^{n}\left[a_{i}, b_{i}\right] \subseteq \mathbb{R}^{n}$.

Here we introduce a piecewise linear parametric approximation for $f(x)$ which is the extension of definition 2.2.

Definition 2.4: Consider the nonlinear smooth function $f():. A \rightarrow \mathbb{R}$ where $A=\prod_{i=1}^{n}\left[a_{i}, b_{i}\right]$. Also consider $P_{M}(A)$ as a partition of $A$ as follows:

$$
P_{M}(A)=\left\{A_{i_{1}, i_{2}, \cdots, i_{n}} \mid i_{1}, \cdots, i_{n}=0,1, \cdots, r-1\right\},
$$

where

$$
A_{i_{1}, i_{2}, \cdots, i_{n}}=\left[\lambda_{i_{1}}, \lambda_{(i+1)_{1}}\right] \times\left[\lambda_{i_{2}}, \lambda_{(i+1)_{2}}\right] \times \cdots \times\left[\lambda_{i_{n}}, \lambda_{(i+1) n}\right](3)
$$

Hence $A$ is partitioned to $M$ cells where $M=r^{n}$. Let for $k=1, \cdots, M$ shown the $k^{\text {th }}$ cell of this partition by $E_{k}$ and $s_{k}=\left(s_{k}^{1}, \cdots, s_{k}^{n}\right)$ is used to shown any point of $E_{k}$. Now $f_{k}(x)$ is defined as a linear parametric approximation of $f(x)$ for $x \in E_{k}$ as follows:

$$
f_{k}(x)=\left.\nabla f(x)\right|_{x=s_{k}} \cdot\left(x-s_{k}\right)+f\left(s_{k}\right)
$$

where $s_{k} \in E_{k}$ for $k=1, \cdots, M$.

Now $G_{M}(x)$ is defined as a pricewise linear approximation of $f(x)$ on $A$ as follows:

$$
G_{M}(x)=\sum_{k=1}^{M}\left[f_{k}(x) \chi_{E_{k}}(x)\right]
$$

Note 2.2: As note 2.1 we have

$$
\lim _{\left\|P_{M}(A)\right\| \rightarrow 0} G_{M}(x)=f(x) .
$$

\section{Description of the Approach}

At first for description our approach we consider the nonlinear programming problems which are shown in (2).
In our approach we introduce the parametric linearization approximation for nonlinear functions. For solving the optimization problem (2) the nonlinear objective function and constraints are transformed approximately to the piecewise linear functions.

We consider $P_{M}(A)$ which is explained above be a partitions of the cell $[0,1]^{n}$. Without loss of generality we can assume that this partition of the region $[0,1]^{n}$ is regular, it means that we have sub-intervals of the form $\left[\lambda_{i_{j}}, \lambda_{i+1_{j}}\right] ; j=1, \cdots, n$ and $\lambda_{i_{j}}=i_{j} h$ where $h=\frac{1}{r}$ and $i_{j}=0,1, \cdots, r-1$.

We consider the nonlinear constraints of the form $x_{j}^{2}-x_{j}=0, j=1, \cdots, n$. By using the parametric linearization approach we may change approximately this constraint to the following form:

$$
\begin{gathered}
\left(2 \bar{\lambda}_{i_{j}}-1\right) x_{j}-\bar{\lambda}_{i_{j}}^{2}=0, x_{j} \in\left[\lambda_{i_{j}}, \lambda_{(i+1)_{j}}\right] \\
j=1, \cdots, n, \bar{\lambda}_{i_{j}}=\frac{\lambda_{i_{j}}+\lambda_{(i+1)_{j}}}{2} .
\end{gathered}
$$

After this according to the following manner in each sub-region of the form $A_{i_{1}, i_{2}, \cdots, i_{n}}$ of $[0,1]^{n}$ we may transform the other nonlinear functions in the optimization problem (2) to the parametric linear approximation form. So each nonlinear functions $f(x) ; g_{s}(x), s=1, \cdots, m$; $h_{k}(x), \quad k=1, \cdots, l$ in the $A_{i_{1}, i_{2}, \cdots, i_{n}}$ sub-regions transformed approximately to the following parametric linear form:

$$
\begin{gathered}
f(x)=f(\bar{\lambda})+\sum_{j=1}^{n} \frac{\partial f(\bar{\lambda})}{\partial x_{j}} x_{j}-\sum_{j=1}^{n} \frac{\partial f(\bar{\lambda})}{\partial x_{j}} \bar{\lambda} \\
g_{s}(x)=g_{s}(\bar{\lambda})+\sum_{j=1}^{n} \frac{\partial g_{s}(\bar{\lambda})}{\partial x_{j}} x_{j}-\sum_{j=1}^{n} \frac{\partial g_{s}(\bar{\lambda})}{\partial x_{j}} \bar{\lambda} \\
s=1, \cdots, m \\
h_{k}(x)=h_{k}(\bar{\lambda})+\sum_{j=1}^{n} \frac{\partial h_{k}(\bar{\lambda})}{\partial x_{j}} x_{j}-\sum_{j=1}^{n} \frac{\partial h_{k}(\bar{\lambda})}{\partial x_{j}} \bar{\lambda} \\
k=1, \cdots, l
\end{gathered}
$$

where $\bar{\lambda}=\left[\bar{\lambda}_{i_{1}}, \bar{\lambda}_{i_{2}}, \cdots, \bar{\lambda}_{i_{n}}\right]$ such that $\bar{\lambda}_{i_{j}}=\frac{\lambda_{i_{j}}+\lambda_{(i+1)_{j}}}{2}$, $j=1, \cdots, n$ and $x=\left(x_{1}, \cdots, x_{n}\right)$ such that $x_{j} \in\left[\lambda_{i_{j}}, \lambda_{(i+1)_{j}}\right]$.

Therefore in each sub-region of the above form we have the following linear programming problem: 


$$
\left\{\begin{array}{ccc}
\operatorname{minimize} & \sum_{j=1}^{n} \frac{\partial f(\bar{\lambda})}{\partial x_{j}} x_{j}+f(\bar{\lambda})-\sum_{j=1}^{n} \frac{\partial f(\bar{\lambda})}{\partial x_{j}} \bar{\lambda} & \\
\text { subject to } & \sum_{j=1}^{n} \frac{\partial g_{s}(\bar{\lambda})}{\partial x_{j}} x_{j}+g_{s}(\bar{\lambda})-\sum_{j=1}^{n} \frac{\partial g_{s}(\bar{\lambda})}{\partial x_{j}} \bar{\lambda} \leq 0 & s=1, \cdots, m \\
\sum_{j=1}^{n} \frac{\partial h_{k}(\bar{\lambda})}{\partial x_{j}} x_{j}+h_{k}(\bar{\lambda})-\sum_{j=1}^{n} \frac{\partial h_{k}(\bar{\lambda})}{\partial x_{j}} \bar{\lambda}=0 & k=1, \cdots, l \\
\left(2 \bar{\lambda}_{i_{j}}-1\right) x_{j}-2 \bar{\lambda}_{i_{j}}^{2}=0 & j=1, \cdots, n \\
x_{j} \in\left[\lambda_{i_{j}}, \lambda_{(i+1)_{j}}\right] & j=1, \cdots, n
\end{array}\right.
$$

For solving the above linear programming problem we divided the interval $[0,1]$ to $r$ equal sub-interval of the form $\left[0, \frac{1}{r}\right] \cup\left[\frac{2}{r}, \frac{3}{r}\right] \cup \cdots \cup\left[\frac{r-1}{r}, 1\right]$. We know that in the solution of the optimization problem (3) all variables must be equal zero or one. Therefore after dividing the interval $[0,1]$ we only should consider the first and the last sub-intervals $\bar{A}_{r}=\left[0, \frac{1}{r}\right]$ and $\tilde{A}_{r}=\left[\frac{r-1}{r}, 1\right]$. In these sub-intervals let $\bar{\lambda}_{i_{j}}=\frac{1}{2 r}$ and $\bar{\lambda}_{i_{j}}=\frac{2 r-1}{2 r}$ so we have $2^{n}$ linear programming problems of the form which be shown in (4). Let this linear programming problems be solved the optimum solution and the optimal value of the objective function of this linear programming problems are shown with $x_{i}^{*}$ and $z_{i}^{*}$ respectively then the optimal solution of the original optimization problem of the form (2) may be calculated as follows:

$$
z^{*}=\min _{1 \leq i \leq 2^{2}}\left\{z_{i}^{*}\right\}
$$

According to the theorems and lemmas which are proved in Section 2, we know that if in any partitions of $[0,1]$ the norm of partitions tends to zero we have $\lim _{R \rightarrow \infty} \bar{A}_{R}=\{0\}$ and $\lim _{R \rightarrow \infty} \tilde{A}_{R}=\{1\}$ Therefore if an arbitrary partition of $[0,1]$ be refined then each linear approximation of nonlinear functions $f(x)$ and $g_{s}(x)$; $s=1, \cdots, m$ and $h_{k}(x) ; k=1, \cdots, l$ tends to the values $f(0), g_{s}(0) ; s=1, \cdots, m ; h_{k}(0) ; k=1, \cdots, l$ and $f(1)$ and $g_{s}(1) ; s=1, \cdots, m$ and $h_{k}(1) ; k=1, \cdots, l$ and constraint of the form $\left(2 \bar{\lambda}_{i_{j}}-1\right) x_{j}-\bar{\lambda}_{i_{j}}^{2}=0 ; j=1, \cdots, n$ tends to values $x_{j}=0$ or $x_{j}=1$ which is the hole feasible solution of the original zero-one non-linear programming problem (2).

\section{Decreasing the Number of Sub-Problems}

According to the described approach for solving zero-one nonlinear programming problems this problems must be converted to a sequence of linear programming problems. In this situation if $n$ the number of variable in the main optimization problem is a large number then we faced with $2^{n}$ sub-problems which must be solved.

According to the following manner this number may be decreased. At first the sub-problems are solved sequentially until the first feasible solution has been determined. For the reminder sub-regions by substituting $x_{j}$ with $\bar{\lambda}_{i_{j}}$ the objective function of the converted linear programming problem was calculated. If the calculate value of the objective functions of this linear programming problem is greater than the objective function of the feasible solution the linear programming problem in this sub-region isn't solved otherwise it be solved.

After this if the solved problem has the feasible solution the value of the objective function of this problem was substituted with the older ones. This manner has been repeated until the sub-problems are ended. The saved value of the objective function and corresponding design variable are the approximated answer for the main zero-one nonlinear programming problems.

\section{Numerical Examples}

Now we give the numerical examples to show the efficiency of our proposed algorithm.

Example 5.1: Consider the following zero-one nonlinear programming problem:

$$
\left\{\begin{array}{cc}
\min \quad z=e^{\left(1-x_{1}\right)}\left(1+\sin \sin \left(\frac{\pi}{2} x_{2}\right)\right)+\left(x_{3}-1\right)^{2} \\
\text { s.t. } & x_{1}^{2}+x_{2}^{2}+x_{3}^{2} \leq 2 \\
2 x_{1}\left(x_{2}+1\right)^{2}+x_{1} x_{3} \leq 3 \\
x_{1}+x_{2}+x_{3} \leq 2 \\
x_{1}, x_{2}, x_{3} \in\{0,1\}
\end{array}\right.
$$


In the first stage we divided the region $[0,1]^{3}$ to $r^{3}$ equal sub-regions. According to our notation in (4) we consider the following sub-regions:

$$
\begin{aligned}
& A_{0_{1}, 0_{2}, 0_{3}}=\left[0, \frac{1}{r}\right] \times\left[0, \frac{1}{r}\right] \times\left[0, \frac{1}{r}\right] \\
& A_{0_{1}, 0_{2}, r-1_{3}}=\left[0, \frac{1}{r}\right] \times\left[0, \frac{1}{r}\right] \times\left[1-\frac{1}{r}, 1\right] \\
& A_{0_{1}, r-1_{2}, 0_{3}}=\left[0, \frac{1}{r}\right] \times\left[1-\frac{1}{r}, 1\right] \times\left[0, \frac{1}{r}\right] \\
& A_{0_{1}, r-1_{2}, r-1_{3}}=\left[0, \frac{1}{r}\right] \times\left[1-\frac{1}{r}, 1\right] \times\left[1-\frac{1}{r}, 1\right] \\
& A_{r-1_{1}, 0_{2}, 0_{3}}=\left[1-\frac{1}{r}, 1\right] \times\left[0, \frac{1}{r}\right] \times\left[0, \frac{1}{r}\right]
\end{aligned}
$$

$$
\begin{aligned}
& A_{r-1_{1}, 0_{2}, r-1_{3}}=\left[1-\frac{1}{r}, 1\right] \times\left[0, \frac{1}{r}\right] \times\left[1-\frac{1}{r}, 1\right] \\
& A_{r-1_{1}, r-1_{2}, 0_{3}}=\left[1-\frac{1}{r}, 1\right] \times\left[1-\frac{1}{r}, 1\right] \times\left[0, \frac{1}{r}\right] \\
& A_{r-1_{1}, r-1_{2}, r-1_{3}}=\left[1-\frac{1}{r}, 1\right] \times\left[1-\frac{1}{r}, 1\right] \times\left[1-\frac{1}{r}, 1\right] .
\end{aligned}
$$

In each sub-region of the above form we choose the element $\bar{\lambda}_{i_{j}} ; j=1,2,3$ of $\bar{\lambda}=\left[\bar{\lambda}_{i_{1}}, \bar{\lambda}_{i_{2}}, \bar{\lambda}_{i_{3}}\right]$ equal to the middle point of each intervals. In the other word we have $\bar{\lambda}_{i_{j}}$ is equal to $\frac{1}{2 r}$ or $\frac{2 r-1}{2 r}$.

So in each sub-region nonlinear $0-1$ programming problem converted approximately to the following linear programming problem:

$$
\left\{\begin{array}{c}
\min \quad z=e^{\left(1-\bar{\lambda}_{i_{1}}\right)}\left(1+\sin \left(\frac{\pi}{2} \bar{\lambda}_{i_{2}}\right)\right)+\left(\bar{\lambda}_{i_{3}}-1\right)^{2}-e^{\left(1-\bar{\lambda}_{i_{1}}\right)}\left(1+\sin \left(\frac{\pi}{2} \bar{\lambda}_{i_{2}}\right)\right)\left(x_{1}-\bar{\lambda}_{i_{1}}\right) \\
\text { s.t. } \\
\frac{\pi}{2}\left(\cos \left(\frac{\pi}{2} \bar{\lambda}_{i_{2}}\right)\right) e^{\left(1-\bar{\lambda}_{i_{1}}\right)}\left(x_{2}-\bar{\lambda}_{i_{2}}\right)+2\left(\bar{\lambda}_{i_{3}}-1\right)\left(x_{3}-\bar{\lambda}_{i_{3}}\right) \\
2 \bar{\lambda}_{i_{1}}\left(\bar{\lambda}_{i_{2}}+1\right)^{2}+\bar{\lambda}_{i_{1}} \bar{\lambda}_{i_{2}}+\left(2\left(\bar{\lambda}_{i_{2}}^{2}+1\right)^{2}+\bar{\lambda}_{i_{3}}^{2}+2 \bar{\lambda}_{i_{1}}\left(x_{1}-\bar{\lambda}_{i_{1}}\right)+2 \bar{\lambda}_{i_{2}}\left(x_{2}-\bar{\lambda}_{i_{2}}\right)+2 \bar{\lambda}_{i_{1}}\right)+4 \bar{\lambda}_{i_{1}}\left(x_{3}-\bar{\lambda}_{i_{2}}\right) \\
x_{1}+x_{2}+x_{3} \leq 2 \\
x_{1}, x_{2}, x_{3} \in A_{i_{1}, i_{2}, i_{3}}
\end{array}\right.
$$

This linear programming problems are solved. The optimum value of the original $0-1$ nonlinear programming problem is calculated according (5). In the following tableau we show the approximate optimal solution for different values of $r$.

\begin{tabular}{ccc}
\hline$r$ & $x^{*}$ & $z^{*}$ \\
\hline 10 & $(0.952657,0,1.04734)$ & 1.05075 \\
100 & $(0.995025,0,1.00497)$ & 1.00501 \\
1000 & & 1.005 \\
\hline
\end{tabular}

Example 5.2: Consider the following 0-1 linear programming problem:

$$
\left\{\begin{array}{cc}
\max & z=3 x_{1}+x_{2}+3 x_{3} \\
\text { s.t. } & -x_{1}+2 x_{2}+x_{3} \leq 4 \\
& 4 x_{2}-3 x_{3} \leq 2 \\
& x_{1}-x_{2}+x_{3} \leq 3 \\
& x_{j}=0 \text { or } 1 j=1,2,3
\end{array}\right.
$$

For solving this problem at first we transform it to the following convex nonlinear programming:

$$
\left\{\begin{array}{cc}
\max & z=3 x_{1}+x_{2}+3 x_{3} \\
\text { s.t. } & -x_{1}+2 x_{2}+x_{3} \leq 4 \\
& 4 x_{2}-3 x_{3} \leq 2 \\
& x_{1}-x_{2}+x_{3} \leq 3 \\
& x_{j}^{2}-x_{j}=0 j=1,2,3
\end{array}\right.
$$

Now using the parametric linearization technique we transform the above nonlinear programming problem to the following sequence of linear programming problems:

$$
\left\{\begin{array}{lc}
\max & z=3 x_{1}+x_{2}+3 x_{3} \\
\text { s.t. } & -x_{1}+2 x_{2}+x_{3} \leq 4 \\
& 4 x_{2}-3 x_{3} \leq 2 \\
& x_{1}-x_{2}+x_{3} \leq 3 \\
& \left(2 \bar{\lambda}_{i_{j}}-1\right) x_{j}-\bar{\lambda}_{i_{j}}^{2}=0, j=1,2,3 \\
& \left(x_{1}, x_{2}, x_{3}\right) \in\left[\lambda_{i_{1}}, \lambda_{(i+1)_{1}}\right] \times\left[\lambda_{i_{2}}, \lambda_{(i+1)_{2}}\right] \times\left[\lambda_{i_{3}}, \lambda_{(i+1)_{3}}\right]
\end{array}\right.
$$


Here we consider $r=10$ and assuming that $\bar{\lambda}_{i_{j}}=0.05$ and $\bar{\lambda}_{i_{j}}=0.95$ from the above sequence of linear programming problem we have 8 linear programming problems for solving. After solving these problems on the sub-feasible region and constitute a set of the approximate optimal solution of the sub-problems, the approximate optimal solution of the original problem is obtained from the following optimization problem:

$$
\max _{1 \leq i \leq 8}\left\{z_{i}^{*}\right\}
$$

where $z_{i}^{*}$ is the optimal vale of the objective function of the sub-problems. Then the approximate optimal solution of the original zero-one linear programming problem is obtained $x^{*}=(1.0028,1.0028,1.0028)$ with the optimal value of the objective function is and $z^{*}=7.0194$.

\section{Conclusions}

In this paper we investigated the optimization technique for solving zero-one mixed nonlinear programming problems. We obtained the global convergence for our approach. Numerical examples are shown the effectiveness of the proposed algorithm. The nonlinear integer programming problems can be transformed to the zero-one nonlinear programming problems and then proposed approach can be used for solved this problems.

\section{References}

[1] P. Hansen, "Method of Nonlinear 0-1 Programming," Annals of Discrete Mathematics, Vol. 5, 1979, pp. 53-70.

[2] J. Mitchell, P. M. Pardalos and M. G. C. Resende, "Interior Point Method for Combinatorial Optimization," In: D. Z. Du and P. Paradalos, Eds., Handbook of Combinatorial Optimization, Vol. 1, 1998, pp. 189-298.

[3] G. L. Nemhauser and L. A. Wolsey, "Integer and Combinatorial Optimization," John Wiley and Sons, New
York, 1988.

[4] P. M. Paradalos, "Continuous Approaches to Discrete Optimization Problems," In: G. Di Pillo and F. Gianessi, Eds., Nonlinear Optimization and Applications, Plenum Publishing, New York, 1996, pp. 313-328.

[5] M. S. Chern and R. H. Jan, "Reliability Optimization Problems with Multiple Constraints," IEEE Transactions on Reliability, 1986, Vol. 35, No. 4, pp. 431-436. doi:10.1109/TR.1986.4335497

[6] K. B. Mira and U. Sharma, "An Efficient Algorithm to Solve Integer Programming Problems Arising in SystemReliability Design," IEEE Transactions on Reliability, Vol. 40, No. 1, 1991, pp. 81-91.

[7] E. L. Lawlev and M. D. Bell, "A Method for Solving Discrete Optimization Problems," Operations Research, Vol. 14, No. 6, 1966, pp. 1098-1112. doi:10.1287/opre.14.6.1098

[8] P. C. Gilmol and R. E. Gomory, "The Theory and Computation of Knapsack Function," Operations Research, Vol. 14, 1966, pp. 1045-1074. doi:10.1287/opre.14.6.1045

[9] E. Balas, "An Additive Algorithm for Solving Linear Programs with 0-1 Variable," Operations Research, Vol. 13, No. 4, 1965, pp. 517-545. doi:10.1287/opre.13.4.517

[10] F. Glover, "Improved Linear Integer Programming Formulations of Nonlinear Integer Problems," Management Science, Vol. 22, No. 4, 1975, pp. 455-460. doi: $10.1287 / \mathrm{mnsc} .22 .4 .455$

[11] M. Oral and O. Kettani, "Reformulating Nonlinear Combinatorial Optimization Problems for Higher Computational Efficiency," European Journal of Operational Research, Vol. 58, No. 2, 1992, pp. 236-249. doi:10.1016/0377-2217(92)90210-Z

[12] M. Oral and O. Kettani, "A Linearization Procedure for Quadratic and Cubic Mixed Integer Problems," Operations Research, Vol. 40, Supplement 1, 1992, pp. s109s116.

[13] P. Hansen and C. Meyer, "Improved Compact Linearizations for the Unconstrained Quadratic 0-1 Minimization Problem," Discrete Applied Mathematics, Vol. 157, 2009, pp. 1267-1290. doi:10.1016/j.dam.2007.12.008 